\title{
Generalization of Fixed Point Results via Iterative Process of F-Contraction
}

\author{
Aftab Hussain ${ }^{1, *}$, Arshad Muhammad ${ }^{2}$ \\ ${ }^{1}$ Department of Basic Sciences, Khwaja Fareed University of Engineering \& Information Technology, Rahim Yar Khan Pakistan \\ ${ }^{2}$ Department of Mathematics, International Islamic University, H-10, Islamabad - 44000, Pakistan \\ *Corresponding author: aftabshh@gmail.com
}

Received January 27, 2017; Revised February 27, 2017; Accepted June 14, 2017

\begin{abstract}
The aim of this paper to discuss generalized iterative process of F-contraction and establish new fixed point theorems in complete metric spaces. As an application of our results, we prove existence and uniqueness of functional equations and system of differential equations. Our results provide extension as well as substantial generalizations and improvements of several well known results in the existing comparable literature.
\end{abstract}

Keywords: metric space, fixed point, F contraction, iterative process

Cite This Article: Aftab Hussain, and Arshad Muhammad, "Generalization of Fixed Point Results via Iterative Process of F-Contraction.” Turkish Journal of Analysis and Number Theory, vol. 5, no. 4 (2017): 132-138. doi: 10.12691/tjant-5-4-3.

\section{Introduction}

In 2012, Wardowski [29] introduce a new type of contractions called F-contraction and proved new fixed point theorems concerning F-contraction. Afterwards Se-celean [28], proved fixed point theorems consisting of F-contractions by Iterated function systems. He generalized the Banach contraction principle in a different way than as it was done by different investigators.

Cosentino et al. [11] established some fixed point results of Hardy-Rogers-type for self-mappings on complete metric spaces or complete ordered metric spaces. Lately, Acar et al. [1] introduced the concept of generalized multivalued $F$-contraction mappings further Altun et al. [2] extended multivalued mappings with $\delta$-Distance and established fixed point results in complete metric space. Sgroi et al. [24] established fixed point theorems for multivalued $F$-contractions and obtained the solution of certain functional and integral equations, which was a proper generalization of some multivalued fixed point theorems including Nadler's. Thereafter, many papers have published on F-contractive mappings in various spaces. For more detail see [1-20] and references therein.

Definition 1 [16] Let $(X, d)$ be a metric space. A mapping $T: X \rightarrow X$ is said to be an $F$ contraction if there exists $\tau>0$ such that

$$
\begin{aligned}
& \forall x, y \in X, d(T x, T y)>0 \\
& \Rightarrow \tau+F(d(T x, T y)) \leq F(d(x, y)),
\end{aligned}
$$

where $F: \mathbb{R}_{+} \rightarrow \mathbb{R}$ is a mapping satisfying the following conditions:
(F1) $F$ is strictly increasing, i.e. for all $x, y \in \mathbb{R}_{+}$such that $x<y, F(x)<F(y)$;

(F2) For each sequence $\left\{a_{n}\right\}_{n=1}^{\infty}$ of positive numbers, $\lim _{n \rightarrow \infty} \alpha_{n}=0$ if and only if $\lim _{n \rightarrow \infty} F\left(\alpha_{n}\right)=-\infty$;

(F3) There exists $k \in(0,1)$ such that $\lim _{\alpha \rightarrow 0^{+}} \alpha^{k} F(\alpha)=0$.

We denote by $\Delta_{F}$, the set of all functions satisfying the conditions (F1)-(F3).

Example 2 [29] Let $F: \mathbb{R}_{+} \rightarrow \mathbb{R}$ be given by the formula $F(\alpha)=\ln \alpha$. It is clear that $F$ satisfied (F1)-(F2)-(F3) for any $k \in(0,1)$. Each mapping $T: X \rightarrow X$ satisfying (1:1) is an F-contraction such that

$$
d(T x, T y) \leq e^{-\tau} d(x, y), \text { for all } x, y \in X, T x \neq T y .
$$

It is clear that for $x, y \in X$ such that $T x=T y$ the inequality $d(T x, T y) \leq e^{-\tau} d(x, y)$, also holds, i.e. $T$ is a Banach contraction.

Example 3 [29] If $F(\alpha)=\ln \alpha+\alpha$, then $F$ satis.es ( $F 1)$ (F3) and the condition (1.1) is of the form

$$
\begin{aligned}
& \frac{d(T x, T y)}{d(x, y)} e^{d(T x, T y)-d(x, y)} \leq e^{-\tau}, \\
& \text { for all } x, y \in X, T x \neq T y .
\end{aligned}
$$

Remark 4 From (F1) and (1.1) it is easy to conclude that every F-contraction is necessarily continuous.

Wardowski [29] stated a modified version of the Banach contraction principle as follows.

Theorem 5 [29] Let $(X, d)$ be a complete metric space and let $T: X \rightarrow X$ be an $F$ contraction. Then $T$ has $a$ 
unique fixed point $x^{*} \in X$ and for every $x \in X$ the sequence $\left\{T^{n} x\right\}_{n \in \mathbb{N}}$ converges to $x^{*}$.

\section{Preliminaries}

We recollect some essential notations, required definitions, and primary results coherent with the literature.

Let $(X, d)$ be a metric space. We denote by $C L(X)$ the class of all nonempty closed subsets of $X$. For a nonempty set $X$, we denote by $N(X)$ the class of all nonempty subsets of $X$. For $A, B \in C L(X)$, define a set

$$
E_{A, B}=\left\{\varepsilon>0: A \subseteq N_{\varepsilon}(B), B \subseteq N_{\varepsilon}(A)\right\} .
$$

The Hausdorff metric $H$ on $C L(X)$ induced by metric $d$ is given as:

$$
H(A, B)=\left\{\begin{array}{l}
\text { inf } E_{A, B} \text { if } E_{A, B} \neq \emptyset \\
\infty \text { if } E_{A, B} \neq \emptyset
\end{array} .\right.
$$

Let $f: X \rightarrow X$ and $T: X \rightarrow C L(X)$. A point $x$ in $X$ is called a fixed point of $T$ if $x \in T x$. The set of all fixed points of $T$ is denoted by $F(T)$. Furthermore, a point $x$ in $X$ is called a coincidence point of $f$ and $T$ if $f x \in T x$. The set of all such points is denoted by $C(f, T)$. If for some point $x$ in $X$, we have $x=f x \in T x$, then a point $x$ is called a common fixed point of $f$ and $T$. We denote set of all common fixed points of $f$ and $T$ by $F(f, T)$.

Definition 6 Let $f: X \rightarrow X$ and $T: X \rightarrow C L(X)$. Let $x_{0}$ be an arbitrary but fixed element in $X$. A sequence $\left(x_{n}\right)$ in $X$ is called $f$ iterative sequence of $T$ starting with $x_{0}$, if $f x_{n} \in T x_{n-1} \forall \geq 1$. Then $D\left(f, T, x_{0}\right)$ $=\left\{\left(f x_{n}\right): f x_{n} \in T x_{n-1}, \forall n \geq 1\right\}$ is called a generalized iterative process of $f$ and $T$ starting at $x_{0}$. Note that $D\left(f, T, x_{0}\right)$ reduces to dynamic process of $T$ starting at $x_{0}$ if $f=I_{X}$ (an identity map on $X$ ) [16]. The generalized iterative process $D\left(f, T, x_{0}\right)$ will simply be written as $\left(f x_{n}\right)$.

Example 7 [16] Consider a Banach space $X=C(I)$ with $a$ norm $\|x\|=\sup _{t \in I}|x(t)|, \quad x \in X \quad$ where $I=[0,1]$. Let $T: X \rightarrow 2^{X}$ be such that for any $x \in X, T X$ is a family of the functions $t \mapsto c \int_{0}^{t} x(s) d s$, where $c \in[0,1]$ i.e.,

$$
(T x) t=\left\{c \int_{0}^{t} x(s) d s: c \in[0,1]\right\}, x \in X
$$

and let $x_{0}(t)=t, \quad t \in[0,1]$. Then the sequence $\left(\frac{1}{n !(n+1) !} t^{n+1}\right)$ is a dynamic process of the operator $T$ starting at $x_{0}$.

Definition 8 Let $f$ be a self map on a metric space $X$. A multivalued mapping $T: X \rightarrow C L(X)$ is called generalized multivalued Ciric type F-contraction with respect to a iterative process $D\left(f, T, x_{0}\right)$ if there exist $F \in \Delta_{F}$ and $\tau: \mathbb{R}_{+} \rightarrow \mathbb{R}_{+}$such that

$$
\begin{aligned}
& \forall_{n \in \mathbb{N}} d\left(f x_{n}, f x_{n+1}\right)>0 \\
& \Rightarrow \tau\left(M\left(x_{n-1}, x_{n}\right)\right)+F\left(d\left(f x_{n}, f x_{n+1}\right)\right) \\
& \quad \leq F\left(M\left(x_{n-1}, x_{n}\right)\right)
\end{aligned}
$$

where

$$
\begin{aligned}
& M\left(x_{n-1}, x_{n}\right) \\
& =\max \left\{\begin{array}{l}
d\left(f x_{n-1}, f x_{n}\right),\left(f x_{n-1}, T x_{n-1}\right), \\
d\left(f x_{n}, T x_{n}\right), \frac{d\left(f x_{n-1}, T x_{n}\right)+d\left(f x_{n}, T x_{n-1}\right)}{2}
\end{array}\right\}
\end{aligned}
$$

and $\forall_{t \geq 0} \liminf _{s \rightarrow t^{+}} \tau(s)>0$.

Example 9 Let $X=[0, \infty), \quad f: X \rightarrow X \quad$ and $T: X \rightarrow C L(X)$ be defined as $f(x)=\frac{x-1}{2}, T X=\left[0, \frac{x}{2}\right]$ for all $x \in X$, we obtain a sequence $f\left(x_{n}\right) \in T\left(x_{n-1}\right)$ $\forall n \geq 1$, these can be many $f$ iterative sequence of $T$ starting at $x_{0}$.

$$
x_{n}=x_{n-1}+1
$$

Let $x_{0}=1$ be an arbitrary point in $X$. Then

$$
\begin{aligned}
& x_{1}=1+x_{0}=2 \\
& f\left(x_{1}\right)=\frac{x_{1}-1}{2}=\frac{1}{2} \in T x_{0}=\left[0, \frac{1}{2}\right] \\
& x_{2}=1+x_{1}=3 \\
& f\left(x_{2}\right)=\frac{x_{2}-1}{2}=1 \in T x_{1}=[0,1] \\
& x_{3}=1+x_{2}=4 \\
& f\left(x_{3}\right)=\frac{x_{3}-1}{2}=\frac{3}{2} \in T x_{2}=\left[0, \frac{3}{2}\right] \\
& x_{4}=1+x_{3}=5 \\
& f\left(x_{4}\right)=\frac{x_{4}-1}{2}=\frac{4}{2}=2 \in T x_{3}=[0,2] \\
& x_{5}=1+x_{4}=6 \\
& f\left(x_{4}\right)=\frac{x_{5}-1}{2}=\frac{5}{2} \in T x_{4}=\left[0, \frac{5}{2}\right]
\end{aligned}
$$

Then clearly $f\left(x_{n}\right) \in T\left(x_{n-1}\right)$. We obtain a generalized iterative process 


$$
\begin{aligned}
& D(f, T, 1) \\
& =\left\{\left(f x_{n}\right)_{n \in \mathbb{N}}: \forall_{n \in \mathbb{N}} f x_{n} \in T x_{n-1}\right\} \\
& =\frac{1}{2}, 1, \frac{3}{2}, 2, \frac{5}{2}, \ldots
\end{aligned}
$$

is called a generalized dynamic process of $f$ and $T$ starting at $x_{0}=1$. So you can construct many $f$ iterative sequences of $T$ starting at $x 0$ for different values.

\section{Main Result}

Throughout this section, we assume that the mapping $F$ is right continuous. In the following we will consider only the dynamic processes $\left(f x_{n}\right)$ satisfying the following condition:

(D) For any $n$ in $\mathbb{N}$,

$$
d\left(f x_{n}, f x_{n+1}\right)>0 \Rightarrow d\left(f x_{n-1}, f x_{n}\right)>0 \text {. }
$$

If dynamic processes $\left(f x_{n}\right)$ does not satisfy property $(D)$, then there exists $n_{0} \in \mathbb{N}$ such that $d\left(f x_{n_{0}}, f x_{n_{0}+1}\right)>0$ and $d\left(f x_{n_{0}-1}, f x_{n_{0}}\right)=0 \quad$ which implies that $f x_{n_{0}-1}=f x_{n_{0}} \in T x_{n_{0}-1}$, that is, the set of coincidence point of hybrid pair $(f, T)$ is nonempty. Under suitable conditions on hybrid pair $(f, T)$, one obtaines the existence of common fixed point of $(f, T)$.

Our main result is the following.

Theorem 10 Let $x_{0} \in X, f: X \rightarrow X$ and $T: X \rightarrow C L(X)$ a generalized multivalued Ciric type $F$-contraction with respect to generalized iterative process $D\left(f, T, x_{0}\right)$. Then $C(f, T) \neq \varnothing$ provided that $f(X)$ is complete and $F$ is continuous or $T$ is closed multivalued mapping. Moreover $F(f, T) \neq \emptyset$ if one of the following conditions holds:

(a) for some $x \in C(f, T)$, $f$ is $T$ - weakly commuting at $x, f^{2} x=f x$. (b) $f(C(f, T))$ is a singleton subset of $C(f, T)$.

Proof. Let $\left(f x_{n}\right)$ be a generalized iterative process of the mapping $f$ and $T$ starting at $x_{0}$. We observe that if there exists $n_{0} \in \mathbb{N}$ such that $x_{n_{0}+1}=x_{n_{0}}$, then the existence of a fixed point is obvious. Hence we can assume that $d\left(x_{n}, x_{n+1}\right)>0$ for all $n \in \mathbb{N}$. Since $T$ is a generalized multivalued Ciric type $F$ - contraction with respect to a generalized iterative process, it follows that

$$
\begin{aligned}
& \tau\left(M\left(x_{n-1}, x_{n}\right)\right)+F\left(d\left(f x_{n}, f x_{n+1}\right)\right) \\
\leq & F\left(M\left(x_{n-1}, x_{n}\right)\right) .
\end{aligned}
$$

implies

$$
\begin{aligned}
& F\left(d\left(f x_{n}, f x_{n+1}\right)\right) \\
& \leq F\left(\max \left\{\begin{array}{l}
d\left(f x_{n-1}, f x_{n}\right), d\left(f x_{n-1}, T x_{n-1}\right), \\
d\left(f x_{n}, T x_{n}\right), \\
\frac{d\left(f x_{n-1}, T x_{n}\right)+d\left(f x_{n}, T x_{n-1}\right)}{2}
\end{array}\right\}\right) \\
& -\tau\left(\max \left\{\begin{array}{l}
d\left(f x_{n-1}, f x_{n}\right), d\left(f x_{n-1}, T x_{n-1}\right), \\
d\left(x_{n}, T x_{n}\right), \\
\frac{d\left(f x_{n-1}, T x_{n}\right)+d\left(f x_{n}, T x_{n-1}\right)}{2}
\end{array}\right\}\right) \\
& \leq F\left(\max \left\{\begin{array}{l}
d\left(f x_{n-1}, f x_{n}\right), d\left(f x_{n-1}, f x_{n}\right), \\
d\left(f x_{n}, f x_{n+1}\right), \\
\frac{d\left(f x_{n-1}, f x_{n+1}\right)+d\left(f x_{n}, f x_{n}\right)}{2}
\end{array}\right\}\right) \\
& -\tau\left(\max \left\{\begin{array}{l}
d\left(f x_{n-1}, f x_{n}\right), d\left(f x_{n-1}, f x_{n}\right), \\
d\left(f x_{n}, f x_{n+1}\right), \\
\frac{d\left(f x_{n-1}, f x_{n+1}\right)+d\left(f x_{n}, f x_{n}\right)}{2}
\end{array}\right\}\right) \\
& \leq F\left(\max \left\{\begin{array}{l}
d\left(f x_{n-1}, f x_{n}\right), d\left(f x_{n}, f x_{n+1}\right), \\
\frac{d\left(f x_{n-1}, f x_{n+1}\right)+d\left(f x_{n}, f x_{n}\right)}{2}
\end{array}\right\}\right) \\
& -\tau\left(\max \left\{\begin{array}{l}
d\left(f x_{n-1}, f x_{n}\right), d\left(f x_{n}, f x_{n+1}\right), \\
\frac{d\left(f x_{n-1}, f x_{n+1}\right)+d\left(f x_{n}, f x_{n}\right)}{2}
\end{array}\right\}\right) \\
& =F\left(\max \left\{d\left(f x_{n-1}, f x_{n}\right), d\left(f x_{n}, f x_{n+1}\right)\right\}\right) \\
& -\tau\left(\max \left\{d\left(f x_{n-1}, f x_{n}\right), d\left(f x_{n}, f x_{n+1}\right)\right\}\right) .
\end{aligned}
$$

Implies that

$$
\begin{aligned}
& F\left(d\left(f x_{n}, f x_{n+1}\right)\right) \\
& \leq F\left(\max \left\{d\left(f x_{n-1}, f x_{n}\right), d\left(f x_{n}, f x_{n+1}\right)\right\}\right) \\
& -\tau\left(\max \left\{d\left(f x_{n-1}, f x_{n}\right), d\left(f x_{n}, f x_{n+1}\right)\right\}\right) .
\end{aligned}
$$

Since $F$ is strictly increasing, therefore

$d\left(f x_{n}, f x_{n+1}\right)<\max \left\{d\left(f x_{n-1}, f x_{n}\right), d\left(f x_{n}, f x_{n+1}\right)\right\}$.

If

$$
\max \left\{d\left(f x_{n-1}, f x_{n}\right), d\left(f x_{n}, f x_{n+1}\right)\right\}=d\left(f x_{n}, f x_{n+1}\right)
$$

for some $n$, then,

$$
d\left(f x_{n}, f x_{n+1}\right)<d\left(f x_{n}, f x_{n+1}\right)
$$

gives a contradiction. So we have

$$
d\left(f x_{n}, f x_{n+1}\right)<d\left(f x_{n-1}, f x_{n}\right) .
$$


Consequently

$$
\begin{aligned}
& \tau\left(d\left(f x_{n-1}, f x_{n}\right)\right)+F\left(d\left(f x_{n}, f x_{n+1}\right)\right) \\
& \leq F\left(d\left(f x_{n-1}, f x_{n}\right)\right) .
\end{aligned}
$$

for all $n \in \mathbb{N}$. By definition 8, there exists $b>0$ and $n \in \mathbb{N}$ such that $\tau\left(d\left(x_{n}, x_{n+1}\right)\right)>b$ for all $n>n_{0}$. Thus, we obtain

$$
\begin{aligned}
& F\left(d\left(f x_{n}, f x_{n+1}\right)\right) \\
\leq & F\left(d\left(f x_{n-1}, f x_{n}\right)\right)-\tau\left(d\left(f x_{n-1}, f x_{n}\right)\right) \\
\leq & F\left(d\left(f x_{n-2}, f x_{n-1}\right)\right)-\tau\left(d\left(f x_{n-2}, f x_{n-1}\right)\right) \\
& -\tau\left(d\left(f x_{n-1}, f x_{n}\right)\right) \\
& \vdots \\
\leq & F\left(d\left(f x_{0}, f x_{1}\right)\right)-\tau\left(d\left(f x_{0}, f x_{1}\right)\right)-\ldots-\tau\left(d\left(f x_{n-1}, f x_{n}\right)\right) \\
= & F\left(d\left(f x_{0}, f x_{1}\right)\right)-\left(\tau\left(d\left(f x_{0}, f x_{1}\right)\right)+\ldots\right. \\
& -\left(\tau\left(d\left(f x_{n_{0}}, f x_{n_{0}+1}\right)\right)+\ldots+\tau\left(d\left(f x_{n_{0}-1}, f x_{n_{0}}\right)\right)\right) \\
\leq & F\left(d\left(f x_{0}, f x_{1}\right)\right)-\left(n-n_{0}\right) b
\end{aligned}
$$

On taking limit as $n \rightarrow \infty$, we have $\lim _{n \rightarrow \infty} F\left(d\left(f x_{n}, f x_{n+1}\right)\right)=-\infty$. By $\quad(F 1), \quad$ we get $\lim _{n \rightarrow \infty} d\left(f x_{n}, f x_{n+1}\right)=0$. By (F3) there exists an $r \in(0,1)$ such that

$$
\lim _{n \rightarrow \infty}\left\{d\left(f x_{n}, f x_{n+1}\right)\right\}^{r} F\left(d\left(f x_{n}, f x_{n+1}\right)\right)=-\infty .
$$

Hence it follows that

$$
\begin{aligned}
& \left\{d\left(f x_{n}, f x_{n+1}\right)\right\}^{r} F\left(d\left(f x_{n}, f x_{n+1}\right)\right) \\
& -\left\{d\left(f x_{n}, f x_{n+1}\right)\right\}^{r} F\left(d\left(f x_{0}, f x_{1}\right)\right) \\
& \leq d\left(f x_{n}, f x_{n+1}\right)^{r}\left[F\left(d\left(f x_{0}, f x_{1}\right)\right)-\left(n_{0}-n\right) b\right] \\
& -d\left(x_{n}, x_{n+1}\right)^{r} F\left(d\left(f x_{0}, f x_{1}\right)\right) \\
& =-\left(n-n_{0}\right) b\left[d\left(f x_{n}, f x_{n+1}\right)\right]^{r} \leq 0 .
\end{aligned}
$$

On taking limit as $n$ tends to $\infty$, we obtain $\lim _{n \rightarrow \infty} n\left\{d\left(f x_{n}, f x_{n+1}\right)\right\}^{r}=0$, that is, $\lim _{n \rightarrow \infty} n^{1 / r} d\left(f x_{n}, f x_{n+1}\right)$ $=0$. This implies that $\sum_{n=1}^{\infty} d\left(f x_{n}, f x_{n+1}\right)$ is convergent and hence the sequence $\left\{f x_{n}\right\}_{n \geq 1}$ is a Cauchy sequence in $f(X)$, so there is $p \in f(X)$ such that $\lim _{n \rightarrow \infty} f x_{n}=p$. Suppose that $u^{*}$ is in $X$ such that $f u^{*}=p$. Next we prove that $f u^{*} \in T u^{*}$. Indeed, assume the contrary, then $d\left(f u^{*}, T u^{*}\right)>0$ because $T u^{*}$ is closed: Since $F$ is strictly increasing, we deduce that

$$
H\left(T x_{n}, T u^{*}\right)<M\left(x_{n}, u^{*}\right)
$$

for all $n \in \mathbb{N}$. Therefore

$$
d\left(f x_{n+1}, T u^{*}\right) \leq H\left(T x_{n}, T u^{*}\right)<M\left(x_{n}, u^{*}\right) .
$$

Since from definition 8 , we have

$\tau\left(M\left(x_{n}, u^{*}\right)\right)+F\left(d\left(f x_{n+1}, T u^{*}\right)\right) \leq F\left(M\left(x_{n}, u^{*}\right)\right)$

for all $n \in \mathbb{N}$.

Next suppose that $F$ is continuous. Since

$$
\lim _{n \rightarrow \infty} d\left(f x_{n}, T u^{*}\right)=d\left(f u^{*}, T u^{*}\right)
$$

we deduce that

$$
\lim _{n \rightarrow \infty} M\left(x_{n}, u^{*}\right)=d\left(f u^{*}, T u^{*}\right) .
$$

so, by continuity of $F$,

$$
\tau\left(d\left(f u^{*}, T u^{*}\right)\right)+F\left(d\left(f u^{*}, T u^{*}\right)\right) \leq F\left(d\left(f u^{*}, T u^{*}\right)\right)
$$

which provides a contradiction. We conclude that $d\left(f u^{*}, T u^{*}\right)=0$, and thus $f u^{*} \in T u^{*}$. Now let (a) holds, that is for $x \in C(f, T), f$ is $T$-weakly commuting at $x$. So we get $f^{2} x \in T f x$. By the given hypothesis $f x=f^{2} x$ and hence $f x=f^{2} x \in T f x$. Consequently $f x \in F(f, T)$. (b) Since $f(C(f, T))=\{x\}$ (say) and $x \in C(f, T)$, this implies that $x=f x \in T x$. Thus $F(f, T) \neq \emptyset$.

Example 11 Let $X=[0,1], \quad f: X \rightarrow X \quad$ and $T: X \rightarrow C L(X)$ be defined as $f(x)=x, \quad T x=\left[0, \frac{x}{2}\right]$ and $d$ be the usual metric on $X$. Define $F: R^{+} \rightarrow R$ and $\tau: \mathbb{R}_{+} \rightarrow \mathbb{R}_{+} \quad$ by $\quad \tau(t)=\left\{\begin{array}{l}-\ln (t+1) \text { for } t \in(0,1) \\ \ln 3 \text { for } t[1, \infty)\end{array}\right.$ and $F(t)=\ln (t)+t$ for all $t>0$. Then for all $x, y \in X$, $T x \neq T y$, we obtain

$$
\begin{aligned}
& \tau(M(x, y))+F(H(T x, T y)) \\
& =\ln (t)+\ln (H(T x, T y))+H(T x, T y) \\
& =\ln (t)+\ln \left(\frac{1}{2}|y-x|\right)+\frac{1}{2}|y-x| \\
& \leq \ln (t)+\ln \left(\frac{1}{t}\right)+\ln \left(\frac{1}{2}|y-x|\right)+\frac{1}{2}|y-x| \\
& \leq \ln (|y-x|)+|y-x| \\
& =F(d(x, y)) \leq F(M(x, y)) .
\end{aligned}
$$

Thus all conditions of above Theorem 10 is satisfied and 0 is a fixed point of $T$.

Example 12 Let $X=[1, \infty)$ be the usual metric space. Define $f: X \rightarrow X, \quad \tau: \mathbb{R}_{+} \rightarrow \mathbb{R}_{+}$and $T: X \rightarrow C L(X)$ 
by $f x=x^{2}$ and $T x=[x+2, \infty)$ for all $x \in X$ and $\tau(t)=\left\{\begin{array}{l}-\ln \left(t+\frac{1}{2}\right) \text { for } t \in(0,1) \\ \ln 3 \text { for } t \in[1, \infty)\end{array}\right.$ and $F(t)=\ln (t)$ for all $t>0$. Note that so $f(X)$ is complete. It is easy to check that for all $x, y \in X$ with $T x \neq T y$ (equivalently with $x \neq y$ ), one has

$$
\tau(M(x, y))+F(H(T x, T y)) \leq F(M(x, y))
$$

So we can apply Theorem 10.

Corollary 13 Let $(X, d)$ be a complete metric space, $x_{0}$ be an arbitrary point in $X$, and $T: X \rightarrow C L(X)$ a multivalued Ciric type F-contraction with respect to dynamic process $D\left(T, x_{0}\right)$, either $F$ is continuous or $T$ is closed multivalued mapping. Then there exists a fixed point of $T$.

\section{(1) Applications}

Decision space and a state space are two basic components of dynamic programming problem. State space is a set of states including initial states, action states and transitional states. So a state space is set of parameters representing different states. A decision space is the set of possible actions that can be taken to solve the problem. These general settings allow us to formulate many problems in mathematical optimization and computer programming. In particular the problem of dynamic programming related to multistage process reduces to the problem of solving functional equations

$$
p(x)=\sup _{y \in D}\left\{g(x, y)+G_{1}(x, y, p(\xi(x, y)))\right\},
$$

for $x \in W$

$$
q(x)=\sup _{y \in D}\left\{g^{\prime}(x, y)+G_{2}(x, y, q(\xi(x, y)))\right\},
$$

for $x \in W$,

where $U$ and $V$ are Banach spaces, $W \subseteq U$ and $D \subseteq V$ and

$$
\begin{gathered}
\xi: W \times D \rightarrow W \\
g, g^{\prime}: W \times D \rightarrow \mathbb{R} \\
G_{1}, G_{2}: W \times D \times \mathbb{R} \rightarrow \mathbb{R},
\end{gathered}
$$

for more details on dynamic programming we refer to $[6,7,8,9,23]$. Suppose that $W$ and $D$ are the state and decision spaces respectively. We aim to give the existence and uniqueness of common and bounded solution of functional equations given in (2.6) and (2.7). Let $B(W)$ denotes the set of all bounded real valued functions on $W$. For an arbitrary $h \in B(W)$, define $\|h\|=\sup _{x \in W}|h(x)|$. Then $(B(W),\|\|$.$) is a Banach space endowed with the$ metric $d$ defined as

$$
d(h, k)=\sup _{x \in W}|h x-k x| .
$$

Suppose that the following conditions hold:

$(C 1): G_{1}, G_{2, g}$, and $g^{\prime}$ are bounded.

(C2): For $x \in W, h \in B(W)$ and $b>0$, define

$$
K h(x)=\sup _{y \in D}\left\{g(x, y)+G_{1}(x, y, h(\xi(x, y)))\right\}(
$$

$J h(x)=\sup _{y \in D}\left\{g^{\prime}(x, y)+G_{2}(x, y, h(\xi(x, y)))\right\}(2.10)$

Moreover assume that $\tau: \mathbb{R}_{+} \rightarrow \mathbb{R}_{+}$and $L \geq 0$ such that for every $(x, y) \in W \times D, \quad h, k \in B(W)$ and $t \in W$ implies

$$
\begin{aligned}
& \left|G_{1}(x, y, h(t))-G_{1}(x, y, k(t))\right| \\
& \leq e^{-\tau(t)}[M(h(t), k(t))]
\end{aligned}
$$

where

$$
\begin{aligned}
M(h(t), k(t)) & \\
=\max \{d(J h(t), J k(t)), d(J k(t), K k(t)), & \\
& d(J h(t), K h(t)), \\
& \left.\frac{d(J h(t), K k(t))+d(J k(t), K k(t))}{2}\right\} .
\end{aligned}
$$

(C3): For any $h \in B(W)$, there exists $k \in B(W)$ such that for $x \in W$

$$
K h(x)=J k(x) .
$$

(C4): There exists $h \in B(W)$ such that

$$
K h(x)=J h(x) \text { implies that } J K h(x)=K J h(x) .
$$

Theorem 14 Assume that the conditions (C1)-(C4) are satisfied. If $J(B(W))$ is a closed convex subspace of $B(W)$, then the functional equations (2.6) and (2.7) have a unique, common and bounded solution.

Proof. Note that $(B(W), d)$ is a complete metric space. By (C1), $J, K$ are self-maps of $B(W)$. The condition (C3) implies that $K(B(W)) \subseteq J(B(W))$. It follows from (C4) that $J$ and $K$ commute at their coincidence points. Let $\lambda$ be an arbitrary positive number and $h_{1}, h_{2} \in B(W)$. Choose $x \in W$ and $y_{1}, y_{2} \in D$ such that

$$
K h_{j}<g\left(x, y_{j}\right)+G_{1}\left(x, y_{j}, h_{j}\left(x_{j}\right)\right)+\lambda,
$$

where $x_{j}=\xi\left(x, y_{j}\right), j=1,2$. Further from (2.9) and (2.10), we have

$$
\begin{aligned}
& K h_{1} \geq g\left(x, y_{2}\right)+G_{1}\left(x, y_{2}, h_{1}\left(x_{2}\right)\right) \\
& K h_{2} \geq g\left(x, y_{1}\right)+G_{1}\left(x, y_{1}, h_{2}\left(x_{1}\right)\right) .
\end{aligned}
$$

Then (2.12) and (2.14) together with (2.11) imply 


$$
\begin{aligned}
& K h_{1}(x)-K h_{2}(x) \\
& <G_{1}\left(x, y_{1}, h_{2}\left(x_{2}\right)\right)-G_{1}\left(x, y_{1}, h_{1}\left(x_{1}\right)\right)+\lambda \\
& \leq\left|G_{1}\left(x, y_{1}, h_{1}\left(x_{1}\right)\right)-G_{1}\left(x, y_{1}, h_{2}\left(x_{2}\right)\right)\right|+\lambda \\
& \leq e^{-\tau(t)}(M(h(t), k(t)))+\lambda .
\end{aligned}
$$

Then (2.12) and (2.13) together with (2.11) imply

$$
\begin{aligned}
& K h_{2}(x)-K h_{1}(x) \\
& \leq G_{1}\left(x, y_{1}, h_{2}\left(x_{2}\right)\right)-G_{1}\left(x, y_{1}, h_{1}\left(x_{1}\right)\right) \\
& \leq\left|G_{1}\left(x, y_{1}, h_{1}\left(x_{1}\right)\right)-G_{1}\left(x, y_{1}, h_{2}\left(x_{2}\right)\right)\right| \\
& \leq e^{-\tau(t)}(M(h(t), k(t))) .
\end{aligned}
$$

From (2.15) and (2.16), we have

$$
\left|K h_{1}(x)-K h_{2}(x)\right| \leq e^{-\tau(t)}(M(h(t), k(t))) .
$$

The inequality (2.17) implies

$$
\begin{aligned}
& d\left(K h_{1}(x)-\right.\left.K h_{2}(x)\right) \leq e^{-\tau(t)}[(M(h(t), k(t)))] . \\
& \tau(t)+\ln \left[d\left(K h_{1}(x)-K h_{2}(x)\right)\right] \\
& \leq \ln [(M(h(t), k(t)))] .
\end{aligned}
$$

Therefore by Theorem 10 , the pair $(K, J)$ has a common fixed point $h^{*}$, that is, $h^{*}(x)$ is unique, bounded and common solution of (2.6) and (2.7).

\section{(1) Application of system of integral equations:}

Now we discuss an application of fixed point theorem we proved in the previous section in solving the system of Volterra type integral equations. Such system is given by the following equations:

$$
\begin{aligned}
& u(t)=\int_{0}^{t} K_{1}(t, s, u(s)) d s+g(t), \\
& w(t)=\int_{0}^{t} K_{2}(t, s, w(s)) d s+f(t) .
\end{aligned}
$$

for $t \in[0, a]$, where $a>0$. We find the solution of the system (2.20) and (2.21). Let $C([0, a], \mathbb{R})$ be the space of all continuous functions defined on $[0, a]$. For $u \in C([0, a], \mathbb{R}), \quad$ define supremum norm as: $\|u\|_{\tau}=\sup _{t \in[0, a]}\left\{u(t) e^{-\tau(t) t}\right\}$, where $\tau: \mathbb{R}_{+} \rightarrow \mathbb{R}_{+}$is taken as a function. Let $C([0, a], \mathbb{R})$ be endowed with the metric

$$
d_{\tau}(u, v)=\sup _{t \in[0, a]}\left\|u(t)-v(t) \mid e^{-\tau(t) t}\right\|_{\tau}
$$

for all $\quad u, v \in C([0, a], \mathbb{R})$. With these setting $C\left([0, a], \mathbb{R},\|.\|_{\tau}\right)$ becomes Banach space.
Now we prove the following theorem to ensure the existence of solution of system of integral equations. For more details on such applications we refer the reader to $[3,21]$.

Theorem 15 Assume the following conditions are satisfied: (i) $K_{1}, K_{2}:[0, a] \times[0, a] \times \mathbb{R} \rightarrow \mathbb{R}$ and $f, g:[0, a] \rightarrow \mathbb{R}$ are continuous;

(ii) Define

$$
\begin{aligned}
& T u(t)=\int_{0}^{t} K_{1}(t, s, u(s)) d s+g(t), \\
& S u(t)=\int_{0}^{t} K_{2}(t, s, u(s)) d s+f(t) .
\end{aligned}
$$

Suppose there exist $\tau: \mathbb{R}_{+} \rightarrow \mathbb{R}_{+}$and $L \geq 0$ such that

$$
\left|K_{1}(t, s, u)-K_{1}(t, s, v)\right| \leq \tau(t) e^{-\tau(t)}[M(u, v)]
$$

for all $t, s \in[0, a]$ and $u, v \in C([0, a], \mathbb{R})$, where

$$
M(u, v)=\max \left\{\begin{array}{l}
|S u(t)-S v(t)|,|S v(t)-T v(t)|, \\
|S u(t)-T u(t)|, \\
\frac{|S u(t)-T v(t)|+|S v(t)-T u(t)|}{2}
\end{array}\right\},
$$

(iii) there exists $u \in C([0, a], \mathbb{R})$ such that $\mathrm{Tu}(t)=\mathrm{Su}(t)$ implies $\mathrm{TSu}(t)=\mathrm{STu}(t)$. Then the system of integral equations given in (2:20) and (2:21) has a solution.

Proof. By assumption (iii)

$$
\begin{aligned}
& |T u(t)-T v(t)| \\
& =\int_{0}^{t}\left|K_{1}(t, s, u(s))-K_{1}(t, s, v(s))\right| d s \\
& \leq \int_{0}^{t} \tau(t) e^{-\tau(t)}\left([M(u, v)] e^{-\tau(t) s}\right) e^{-\tau(t) s} d s \\
& \leq \int_{0}^{t} \tau(t) e^{-\tau(t)}\|M(u, v)\|_{\tau} e^{\tau(t) s} d s \\
& \leq \tau(t) e^{-\tau(t)}\|M(u, v)\|_{\tau} \int_{0}^{t} e^{\tau(t) s} d s \\
& \leq \tau(t) e^{-\tau(t)}\|M(u, v)\|_{\tau} \frac{1}{\tau(t)} e^{\tau(t) t} \\
& \leq e^{-\tau(t)}\|M(u, v)\|_{\tau} e^{\tau(t) t} .
\end{aligned}
$$

This implies

$$
|T u(t)-T v(t)| e^{-\tau(t) t} \leq e^{-\tau(t)}\|M(u, v)\|_{\tau},
$$

That is

$$
\|T u(t)-T v(t)\|_{\tau} \leq e^{-\tau(t)}\|M(u, v)\|_{\tau}
$$


which further implies

$$
\tau(t)+\ln \|T u(t)-T v(t)\|_{\tau} \leq \ln \|M(u, v)\|_{\tau} .
$$

So all the conditions of Theorem 10 are satisfied. Hence the system of integral equations given in (2.20) and (2.21) has a unique common solution.

\section{Conclusion}

This paper presents fixed point theorems for generalized iterative process under the improved notion of dynamic process. The presented theorem provide extension as well as substantial generalizations and improvements of several well known results. The present version of these results make significant and useful contribution in the existing literature.

\section{Conflict of Interests}

The authors declare that they have no competing interests.

\section{References}

[1] Ö. Acar, G. Durmaz and G. Minak, Generalized multivalued F-contractions on complete metric spaces, Bulletin of the Iranian Mathematical Society. 40(2014), 1469-1478.

[2] Ö. Acar and I. Altun, A Fixed Point Theorem for Multivalued Mappings with $\delta$-Distance, Abstr. Appl. Anal., Volume 2014, Article ID 497092, 5 pages.

[3] A. Augustynowicz, Existence and uniqueness of solutions for partial differential-functional equations of the first order with deviating argument of the derivative of unknown function, Serdica Mathematical Journal 23 (1997) 203-210.

[4] M. Arshad, Fahimuddin, A. Shoaib and A. Hussain, Fixed point results for $\alpha-\psi$ - -locally graphic contraction in dislocated qusai metric spaces, Math Sci., (2014), 7 pages.

[5] S.Banach, Sur les opérations dans les ensembles abstraits et leur application aux equations itegrales, Fund. Math., 3 (1922) 133-181.

[6] R. Baskaran, P.V. Subrahmanyam, A note on the solution of a class of functional equations. Appl. Anal. 22(3-4), 235-241.

[7] R. Bellman, Methods of Nonlinear Analysis. Vol. II. Mathematics in Science and Engineering, vol. 61. Academic Press, New York (1973)

[8] R. Bellman, E.S. Lee, Functional equations in dynamic programming. Aequ. Math. 17, 1-18 (1978).

[9] P.C. Bhakta, S. Mitra, Some existence theorems for functional equations arising in dynamic programming. J. Math. Anal. Appl. 98, 348-362 (1984).
[10] LB. Ćirić, A generalization of Banach.s contraction principle. Proc. Am. Math. Soc., 45, (1974) 267-273.

[11] M. Cosentino, P. Vetro, Fixed point results for F-contractive mappings of Hardy-Rogers-Type, Filomat 28:4(2014), 715-722.

[12] M. Edelstein, On fixed and periodic points under contractive mappings. J. Lond. Math. Soc., 37, 74-79 (1962).

[13] B. Fisher, Set-valued mappings on metric spaces, Fundamenta Mathematicae, 112 (2) (1981) 141-145.

[14] N. Hussain and P. Salimi, suzuki-wardowski type fixed point theorems for $\alpha$-GF-contractions, Taiwanese J. Math., 20 (20) (2014).

[15] N. Hussain, P Salimi and A. Latif, Fixed point results for single and set-valued $\alpha-\eta-\psi$-contractive mappings, Fixed Point Theory Appl. 2013, 2013: 212.

[16] D. Klim and D.Wardowski, Fixed points of dynamic processes of set-valued $F$-contractions and application to functional equations, Fixed Point Theory Appl., (2015) 2015: 22.

[17] E. Karapinar and B. Samet, Generalized $(\alpha-\psi)$ contractive type mappings and related fixed point theorems with applications, Abstr. Appl. Anal., (2012) Article id: 793486.

[18] MA. Kutbi, W. Sintunavarat, On new fixed point results for $(\alpha, \psi, \xi)$-contractive multi-valued mappings on $\alpha$-complete metric spaces their consequences, Fixed Point Theory Appl., (2015) 2015: 2.

[19] MA. Kutbi, M. Arshad and A. Hussain, On Modified $\alpha-\eta$-Contractive mappings, Abstr. Appl. Anal., Volume 2014, Article ID 657858, 7 pages.

[20] SB. Nadler, Multivalued contraction mappings, Pac. J. Math., 30 (1969), 475-488.

[21] D. ÓRegan, A. Petruşel, Fixed point theorems for generalized contractions in ordered metric spaces, Journal of Mathematical Analysis and Applications 341 (2008) 1241-1252.

[22] H. Piri and P. Kumam, Some fixed point theorems concerning $F$ - contraction in complete metric spaces, Fixed Poin Theory Appl. 2014, 2014: 210.

[23] H.K. Pathak, Y.J. Cho, S.M. Kang, B.S. Lee, Fixed point theorems for compatible mappings of type $\mathrm{P}$ and applications to dynamic programming. Matematiche 50, 15-33 (1995).

[24] M. Sgroi and C. Vetro, Multi-valued F-contractions and the solution of certain functional and integral equations, Filomat 27:7 (2013), 1259-1268.

[25] P. Salimi, A. Latif and N. Hussain, Modified $(\alpha-\psi)$-Contractive mappings with applications, Fixed Point Theory Appl. (2013) 2013: 151.

[26] M. Sgroi, C. Vetro, Multi-valued F-contractions and the solution of certain functional and integral equations, Filomat 27: 7(2013), 1259-1268.

[27] B. Samet, C. Vetro and P. Vetro, Fixed point theorems for $\alpha-\psi$-contractive type mappings, Nonlinear Anal. 75 (2012) 2154-2165.

[28] NA. Secelean, Iterated function systems consisting of F-contractions, Fixed Point Theory Appl. 2013, Article ID 277 (2013).

[29] D. Wardowski, Fixed point theory of a new type of contractive mappings in complete metric spaces. Fixed Poin Theory Appl. 2012, Article ID 94 (2012). 